\section{Reprodutibilidade, consistência interna e validade de construto do KIDSCREEN-27 em adolescentes brasileiros}

\author{
Reproducibility, internal consistency, and \\ construct validity of KIDSCREEN-27 in \\ Brazilian adolescents
}

\section{Reproducibilidad, consistencia interna y validez de constructo del KIDSCREEN-27 en adolescentes brasileños}

\begin{abstract}
Resumo
O objetivo deste trabalho foi estimar reprodutibilidade, consistência interna e validade de construto do KIDSCREEN-27, questionário para mensurar a qualidade de vida relacionada à saúde, em adolescentes brasileiros. Foi realizado um estudo para estimar a reprodutibilidade (176 adolescentes, 59,7\% do sexo feminino, 64,7\% de 10-12 anos de idade) e outro para estimar a consistência interna e a validade (1.321 adolescentes, 53,7\% do sexo feminino, $56,9 \%$ de 10-12 anos de idade). Os estudos foram realizados com adolescentes de ambos os sexos, de escolas públicas, no Município de João Pessoa, Paraíba, Brasil. O KIDSCREEN-27 contém 27 itens distribuidos em cinco domínios (bem-estar físico, 5 itens; bem-estar psicológico, 7 itens; pais e suporte social, 7 itens; autonomia e relação com os pais, 4 itens; ambiente escolar, 4 itens). Para estimar a reprodutibilidade, recorreu-se ao coeficiente de correlação intraclasse - CCI. A análise fatorial confirmatória foi utilizada para avaliar a validade de construto, e o indice de fidedignidade combinada (IFC) para verificar a consistência interna do questionário. Os CCI foram iguais ou superiores a 0,70 (0,70-0,96). As cargas fatoriais foram superiores a 0,40 , exceto para cinco itens $(0,28-0,39)$. Os indicadores de qualidade de ajuste do modelo foram adequados $\left(\chi^{2} / d f=2,79 ; R M R=0,035 ; R M S E A=0,037 ; G F I=0,951\right.$; $A G F I=0,941$; $C F I=0,908$; $T L I=0,901)$. O IFC variou de 0,65 a 0,70 nos domínios e foi igual a 0,90 para o questionário. O KIDSCREEN-27 alcançou níveis satisfatórios de reprodutibilidade, consistência interna e validade de construto, podendo ser utilizado para avaliar a qualidade de vida relacionada à saúde em adolescentes de 10-15 anos de idade.
\end{abstract}

Inquéritos e Questionários; Reprodutibilidade dos Testes;

Qualidade de Vida; Adolescente
QUESTÕES METODOLÓGICAS

METHODOLOGICAL ISSUES

José Cazuza de Farias Júnior 1

Mathias Roberto Loch 2

Antônio José de Lima Neto 3

Joana Marcela Sales 1

Flávia Emília Leite de Lima Ferreira 1

doi: 10.1590/0102-311X00131116

Correspondência

J. C. Farias Júnior

Departamento de Educação Física, Universidade Federal da Paraíba.

Cidade Universitária, Campus I, João Pessoa, PB 58051-900, Brasil.

jcazuzajr@hotmail.com

1 Universidade Federal da Paraíba, João Pessoa, Brasil.

2 Universidade Estadual de Londrina, Londrina, Brasil.

3 Associação Caruaruense de Ensino Superior e Técnico,

Caruaru, Brasil. 


\section{Introdução}

A qualidade de vida relacionada à saúde é um construto subjetivo e multidimensional, envolvendo aspectos gerais da qualidade de vida - bem-estar físico, emocional, social e espiritual - e que claramente podem influenciar os níveis de saúde ${ }^{1}$. Tem sido crescente o interesse em avaliar esse construto em adolescentes, por se tratar de uma fase em que ocorrem mudanças fisiológicas, psicológicas, cognitivas e sociais importantes, que podem influenciar a qualidade de vida 2.

A medida de qualidade de vida relacionada à saúde tem sido utilizada para avaliar e monitorar os níveis de saúde, o impacto de intervenções para a promoção da saúde, na prática clínica e identificar seus fatores correlatos e/ou determinantes 3 . Isso pode explicar o aumento no número de estudos de validação de instrumentos para mensurar a qualidade de vida relacionada à saúde em adolescentes nas últimas duas décadas 4,5. Em geral, esses instrumentos abordam domínios que compreendem aspectos físicos, psicológicos, ambientais e sociais 4,5. Alguns são de uso restrito ou exigem autorização prévia para a sua utilização, inclusive para fins de pesquisa, resultando em aumento nos custos financeiros e limitando o número de aplicações; outros, são destinados aos adolescentes em condições especiais de saúde 6 . Fatores como ser de domínio público e aplicado em um número elevado de pessoas tornam o instrumento mais adequado para avaliar a qualidade de vida relacionada à saúde em estudos epidemiológicos com um grande número de pessoas.

Dentre os instrumentos disponíveis na literatura, o KIDSCREEN (versão com 52, 27 e 10 itens) tem sido um dos mais utilizados 7. Ele foi criado por meio do projeto Screening and Promotion for Health-Related Quality of Life in Children and Adolescents - A European Public Health Perspective, entre os anos de 2001 e 2004, contando com a participação de vários países da Europa 7, com o objetivo de mensurar a qualidade de vida relacionada à saúde em adolescentes, de modo transcultural, sendo de acesso livre e de fácil aplicação 8 .

A versão longa do KIDSCREEN contém 52 itens, distribuídos em 10 domínios 8, e sua validade já foi amplamente avaliada em crianças e adolescentes de diferentes continentes 9 , incluindo a América do Sul 10, tendo alcançado níveis satisfatórios de fidedignidade e validade. No Brasil, esse instrumento foi submetido a um processo de adaptação transcultural (tradução, retrotradução, reprodutibilidade, consistência interna e validade), em adolescentes de 14-19 anos de idade de uma escola de educação básica no Município de Londrina, Paraná 10. Todos os itens saturaram nos domínios propostos na versão original do instrumento, as cargas fatoriais foram superiores a 0,50 , a consistência interna variou de 0,80-0,90, e a reprodutibilidade foi superior a 0,70, indicando boa fidedignidade e validade 10 .

Entretanto, a utilização do KIDSCREEN-52 em estudos epidemiológicos, com grande número de pessoas e de variáveis a serem mensuradas, torna-se praticamente inviável devido ao elevado número de itens que deverão ser respondidos. Nesse sentido, o KIDSCREEN com 27 itens foi desenvolvido para atender a essa finalidade 7 . Essa versão considera os principais domínios ligados à qualidade de vida relacionada à saúde e compreende 27 dos 52 itens da versão longa, distribuídos em cinco domínios: (1) saúde e bem-estar físico; (2) bem-estar psicológico; (3) autonomia e relação com os pais; (4) suporte social e grupo de pares; (5) ambiente escolar. A versão com 27 itens manteve três dos mesmos domínios da versão longa (KIDSCREEN-52): bem-estar físico, apoio social e pares, ambiente escolar; seis domínios foram agrupados em dois: bem-estar psicológico, autonomia e relação com os pais; e um foi retirado: bullying 7. Esse instrumento foi avaliado em adolescentes da Europa 7, Ásia 9,11 e América do Sul 12,13,14, apresentando níveis elevados de fidedignidade e validade.

Apesar de o KIDSCREEN-27 ser composto por 27 dos 52 itens da versão longa, ele tem uma composição dimensional diferente, e sua fidedignidade e validade ainda não foram avaliadas em adolescentes brasileiros. Outro aspecto a ser considerado é o de que a qualidade de vida relacionada à saúde, para além de indicadores do estilo de vida (por exemplo, atividade física, hábitos alimentares, fumo), é influenciada por fatores demográficos, sociais, culturais, ambientais e econômicos 3,15. Um instrumento com níveis adequados de reprodutibilidade, consistência interna e validade para adolescentes de determinado país e/ou região não terá, necessariamente, o mesmo desempenho em jovens com características distintas daquelas dos jovens nos quais ele foi desenvolvido e/ou testado 16,17. Sendo assim, este estudo estimou reprodutibilidade, consistência interna e validade de construto do KIDSCREEN-27 em adolescentes brasileiros. 


\section{Métodos}

Este trabalho faz parte de um projeto amplo denominado Estudo LONCAAFS (Estudo Longitudinal sobre Comportamento Sedentário, Atividade Física, Alimentação e Saúde dos Adolescentes), aprovado pelo Comitê de Ética em Pesquisa com Seres Humanos da Universidade Federal da Paraíba (protocolo no 024/13). A primeira etapa desse projeto consistiu no desenvolvimento e validação dos instrumentos para serem utilizados em sua coleta de dados ao longo dos quatro anos do estudo (2014-2017).

Para atender a uma dessas finalidades, dois estudos foram realizados: um para avaliar a reprodutibilidade; e outro, a consistência interna e a validade de construto do KIDSCREEN-27. Ambos foram desenvolvidos com escolares do Ensino Fundamental II de escolas da rede pública do Município de João Pessoa, Paraíba, Brasil.

\section{Estudo de reprodutibilidade}

Para estimar a reprodutibilidade do questionário foi realizado um estudo com uma amostra de escolares dos 60 e 80 anos do Ensino Fundamental II. Na determinação do tamanho da amostra, considerou-se um coeficiente de correlação intraclasse $(\mathrm{CCI}) \geq 0,20$ (reprodutibilidade), duas aplicações do questionário, erro tipo I de $5 \%$, erro tipo II de $20 \%$ (poder de $80 \%$ ) e acréscimo de $30 \%$ para compensar perdas e recusas. Isso resultou em uma amostra de 218 adolescentes, mas foram convidados 247 para participar do trabalho.

A amostra foi selecionada por conglomerados em dois estágios. No primeiro, foram selecionadas sistematicamente oito escolas, distribuídas proporcionalmente por tipo (estadual, municipal) e região do município (norte, sul, leste, oeste). No segundo, foram selecionadas aleatoriamente 15 turmas, distribuídas proporcionalmente por série do Ensino Fundamental II (6o e 8o anos).

As seguintes informações foram coletadas para caracterizar a amostra estudada: sexo (masculino e feminino), idade (mensurada de forma centesimal e categorizada em 10-12 e 13-14/15 anos de idade) e classe econômica (Metodologia da Associação Brasileira das Empresas de Pesquisa, ABEP 18, que agrupa as pessoas assim: A1 [superior], A2, B1, B2, C1, C2, D e E [inferior], reagrupadas em classes A/B, C e D/E).

Para estimar a reprodutibilidade, o KIDSCREEN-27 foi aplicado (entrevista face a face, tempo médio de aplicação de cinco minutos) em duplicata, com intervalo de dois dias entre as réplicas de aplicação. Optou-se por utilizar esse intervalo de tempo tendo em vista que a semana de referência adotada para os adolescentes responderem às questões do KIDSCREEN-27 foi a anterior à da coleta de dados. Sendo assim, era necessário que os adolescentes recordassem do mesmo período para responder às questões na réplica de aplicação do questionário. Não há consenso na literatura sobre o intervalo de tempo ideal a ser adotado entre as réplicas de aplicação para estimar a reprodutibilidade teste-reteste; essa definição dependerá inclusive da característica da variável que está sendo investigada. Tem sido recomendado que esse intervalo não seja longo, para evitar que possíveis mudanças no fenômeno que está sendo mensurado sejam interpretadas como um erro de medida (podendo resultar em subestimação da reprodutibilidade), nem curto demais, para evitar que os resultados sejam contaminados pelo efeito recordatório (levaria à superestimação da reprodutibilidade) 19.

A coleta de dados foi realizada entre agosto e outubro de 2013, por equipe treinada e submetida previamente a estudo piloto, com amostra contendo as mesmas características das deste estudo, seguindo protocolo uniforme de coleta.

O KIDSCREEN-27 contém 27 itens distribuídos em cinco domínios: (1) saúde e bem-estar físico (5 itens); (2) bem-estar psicológico ( 7 itens); (3) autonomia e relação com os pais (7 itens); (4) suporte social e grupo de pares ( 4 itens); (5) ambiente escolar (4 itens). Todos os itens estavam ancorados por escala Likert (nunca $=1$, raramente $=2$, frequentemente $=3$, sempre $=4$ ). Foram produzidos escores de qualidade de vida relacionada à saúde seguindo os procedimentos previamente descritos 7,8 .

\section{Estudo de validade de construto e consistência interna}

A validade de construto é considerada a forma mais fundamental de validade dos instrumentos psicológicos, constituindo uma maneira direta para se verificar a hipótese da legitimidade da representação 
comportamental dos traços latentes do construto ${ }^{20}$. A análise fatorial representa uma das formas de demonstração da validade de construto de um questionário ou escala para mensurar um determinado fenômeno 20 .

A validade de construto e a consistência interna do questionário foram estimadas em uma amostra representativa de escolares do 6o ano do Ensino Fundamental II de João Pessoa. O tamanho da amostra foi determinado para um estudo de prevalência: população de referência igual a 9.520 escolares; prevalência do desfecho de 50\%; intervalo de 95\% de confiança (IC95\%); erro máximo aceitável de quatro pontos percentuais; efeito de desenho (deff) igual a dois. O tamanho mínimo da amostra ficou estabelecido em 1.130 adolescentes. Foram acrescidos $40 \%$ a esse quantitativo para compensar as possíveis perdas e recusas, resultando em uma amostra com 1.582 adolescentes. Essa amostra é superior ao mínimo necessário para a análise fatorial confirmatória, considerando um destes dois critérios: a amostra deve ter $\geq 500$ participantes ou razão de 10:1 entre o número de participantes na amostra e o de parâmetros livres a serem estimados no modelo 21 . Neste estudo, o número de parâmetros livres para serem estimados no modelo foi igual a $67(67$ x 10 participantes para cada parâmetro $=670$ participantes). Sendo assim, o tamanho da amostra atende aos dois critérios previamente descritos.

A seleção da amostra foi por conglomerado em estágio único, com seleção sistemática de 28 escolas (14 municipais e 14 estaduais), distribuídas proporcionalmente por tamanho (número de alunos matriculados no 6o ano) e região do município (norte, sul, leste, oeste). Nas escolas selecionadas, todos os escolares regularmente matriculados no 6o ano foram convidados para participar do trabalho. A coleta de dados foi realizada entre fevereiro e dezembro de 2014, pela mesma equipe do estudo de reprodutibilidade.

\section{Tabulação e análise dos dados}

Os dados foram tabulados em duplicata no programa EpiData 3.1 (Epidata Association, Odense, Dinamarca), com checagem automática de consistência e amplitude dos valores. A ferramenta "validar dupla digitação" foi utilizada para identificar possíveis erros de digitação. As análises estatísticas foram realizadas no programa Stata 14.1 (StataCorp LP, College Station, Estados Unidos), sendo considerada a estratégia de seleção da amostra (utilização da opção svy deste programa). O nível de significância adotado foi inferior a $5 \%(\mathrm{p}<0,05)$.

O CCI foi usado para avaliar a reprodutibilidade. Valores de CCI iguais ou superiores a 0,70 foram considerados satisfatórios 22 . A análise fatorial confirmatória foi utilizada para avaliar a qualidade de ajuste do modelo e comparar os modelos concorrentes (validade de construto). Para tanto, recorreu-se à Análise de Equações Estruturais, e os parâmetros do modelo foram estimados pelo método da Máxima Verossimilhança (ML - Maximum Likelihood, função de ligação logit), baseado no procedimento de Bootstrap, tendo em vista que os dados não apresentaram distribuição normal multivariada - coeficiente padronizado de Mardia > 5 21. Esse procedimento tem sido recomendado 23,24 e aplicado em estudos similares 7,25 , considerando-se que suas estimativas são robustas e não afetadas pela ausência de distribuição normal multivariada 21,24. Todos os itens do questionário apresentaram valores de assimetria $(|\mathrm{sk}| 0,195$ a 1,755$)$ e curtose $(|\mathrm{ku}| 0,002$ a 1,377$)$ que não se afastam dos considerados adequados para assumir a pressuposição de distribuição normal ( $\mid$ sk $\mid>2-3$ e curtose $|\mathrm{ku}|>7-10) 21$.

A avaliação da qualidade de ajuste dos modelos foi efetuada por meio de diferentes índices de ajustes: qui-quadrado $\left(\chi^{2}\right)$ com valores de $\mathrm{p}>0,05$ ou $\chi^{2} / \mathrm{df} \leq 3$, Root Mean Square Residual - RMR (< 0,05, toleram-se valores até 0,08), Root Mean Square of Approximation - RMSEA $(\leq 0,05$, toleram-se valores até $<0,10)$, Adjusted Goodness of Fit Index - AGFI $(\geq 0,90)$, Goodness of Fit Index - GFI $(\geq 0,90)$, Comparative Fit Index - CFI $(\geq 0,90)$ e Tucker-Lewis Index - TLI $(\geq 0,90) 21$.

O modelo inicial considerou o KIDSCREEN-27 conforme a proposta original. As modificações no modelo foram efetuadas com base no índice de modificação (Modification Indice - MI) e nos valores dos resíduos. O índice de modificação indica as mudanças esperadas nos valores da estatística $\chi^{2}$, caso determinado parâmetro que está fixado seja estimado livremente. Em relação aos valores dos resíduos padronizados, recomenda-se que fiquem em torno de $\pm 2,58$ 21. A análise fatorial confirmatória foi repetida após cada modificação do modelo para avaliar as alterações na qualidade do ajuste.

Analisou-se a consistência interna por meio do índice de fidedignidade combinada, IFC $=\left\{\left[\left(\sum\right.\right.\right.$ das cargas fatoriais $\left.)^{2} /\left(\sum \text { das cargas fatoriais }\right)^{2}\right]+\left[\sum\left(1-\right.\right.$ cargas fatoriais $\left.\left.\left.^{2}\right)\right]\right\}$. Optou-se pelo IFC dado que 
os valores de alfa de Cronbach $(\alpha)$ podem ser subestimados em escalas com dois ou mais domínios. Valores do IFC foram considerados satisfatórios quando $\geq 0,7026$.

\section{Resultados}

Dos 247 adolescentes convidados para participar do estudo de reprodutibilidade, quatro se recusaram ou não foram autorizados pelos pais, 67 foram excluídos ( 8 com idades $<10$ ou $>15$ anos e 59 ausentes na segunda aplicação do questionário) - a análise de reprodutibilidade envolveu 176 adolescentes de 10-15 anos de idade. No estudo de validade, dos 1.475 que responderam ao questionário, foram excluídos 154 adolescentes que não tinham dados completos para um ou mais itens do questionário de qualidade de vida relacionada à saúde - foram analisados dados completos de 1.321 adolescentes de 10-14 anos de idade. Não houve diferenças significativas $(\mathrm{p} \geq 0,05)$ para as características sociodemográficas, atividade física e excesso de peso corporal dos adolescentes incluídos e excluídos das análises.

As características sociodemográficas da amostra de cada estudo estão apresentadas na Tabela 1. A maioria dos adolescentes era do sexo feminino, tinha de 10-12 anos de idade, com cor da pele não branca e era de classe econômica de média a baixa. Os valores médios dos escores de qualidade de vida variaram de 75,6 a 84,0 pontos. As variáveis analisadas não apresentaram diferenças estatisticamente significativas $(\mathrm{p} \geq 0,05)$ entre as duas amostras estudadas.

Em relação à reprodutibilidade, os valores de CCI variaram de 0,75 (domínio 1 [D1] - bem-estar físico) a 0,89 (domínio 5 [D5] - ambiente escolar) e foi igual a 0,90 para o escore geral do questionário. Pequenas variações nos valores CCI foram observadas nos subgrupos analisados (sexo, faixa etária, classe econômica) quando comparados aos valores para todos os adolescentes (Tabela 2).

Os resultados da análise fatorial confirmatória demonstraram que as cargas fatoriais (CF) foram superiores a 0,40, exceto para os itens "PAF - praticou atividades físicas" ( $\mathrm{CF}=0,39)$, no domínio 1 (D1) - bem-estar físico; "ST - você se sentiu tão triste" (CF =0,32), "SM - você se sentiu tão mal que não queria fazer nada" (CF = 0,28) e "SS - você se sentiu sozinho" (CF =0,33), no domínio 2 (D2) bem-estar psicológico; e o item "DA - teve dinheiro suficiente para fazer as mesmas coisas que seus amigos fizeram” (CF = 0,39), no domínio 3 (D3) - pais e suporte social (Figura 1).

Os indicadores da qualidade de ajuste do modelo mostraram que a estrutura fatorial do KIDSCREEN-27 alcançou níveis aceitáveis $\left(\chi^{2}[\mathrm{df}]=1098,4\right.$ [314], $\mathrm{p}<0,001 ; \chi^{2} / \mathrm{df}=3,49 ; \mathrm{RMR}=0,040$; RMSEA = 0,044 [IC90\%: 0,041-0,046]; GFI = 0,938; AGFI =0,926; CFI =0,870; TLI = 0,854). Entretanto, com base nos índices de modificações de ajuste e dos valores dos resíduos, o modelo original do KIDSCREEN-27 foi reespecificado, sendo adicionadas covariâncias entre os erros dos seguintes itens: ST - "você se sentiu triste", SM - "você se sentiu tão mal que não queria fazer nada", SS - "você se sentiu sozinho", DA - "teve dinheiro suficiente para fazer as mesmas coisas que seus amigos fizeram" e DS - "teve dinheiro suficiente para os seus gastos". Esse modelo apresentou melhores indicadores de qualidade de ajuste $\left(\chi^{2}[\mathrm{df}]=866,3\right.$ [311], $\mathrm{p}<0,001 ; \chi^{2} / \mathrm{df}=2,79 ; \mathrm{RMR}=0,035 ; \mathrm{RMSEA}=0,037$ [IC90\%: 0,034-0,040]; GFI = 0,951; AGFI = 0,941; CFI =0,908; TLI =0,901) comparativamente ao que não continha covariâncias entre os erros. Verificou-se que a qualidade de ajuste desse modelo foi similar em todos os subgrupos analisados (sexo, faixa etária, classe econômica) (Tabela 3).

A medida de consistência interna (IFC) variou de 0,65 (domínios 1, 2, 4 e 5) a 0,70 (domínio 3) nos domínios, e foi igual a 0,90 para o questionário. Pequenas variações foram observadas nos valores do IFC nos subgrupos analisados (Tabela 4).

\section{Discussão}

Este trabalho demonstrou que o modelo original do KIDSCREEN-27 alcançou níveis satisfatórios de reprodutibilidade, consistência interna e validade de construto, mostrando-se adequado para mensurar a qualidade de vida relacionada à saúde em adolescentes brasileiros de 10-15 anos de idade.

Dentre os estudos que analisaram as propriedades psicométricas do KIDSCREEN-27, poucos avaliaram a sua reprodutibilidade. Ravens-Sieberer et al. 7 , em estudo com adolescentes de 8 a 18 anos de 13 países da Europa (CCI: 0,61 a 0,74), e Andersen et al. 27, em pesquisa com adolescentes da 
Tabela 1

Descrição das características da amostra do estudo de reprodutibilidade, consistência interna e validade de construto do questionário de qualidade de vida relacionado à saúde - KIDSCREEN-27 em adolescentes de João Pessoa, Paraíba, Brasil.

\begin{tabular}{|c|c|c|c|c|}
\hline \multirow[t]{2}{*}{ Variáveis } & \multicolumn{2}{|c|}{$\begin{array}{l}\text { Estudo de reprodutibilidade } \\
\qquad(\mathrm{n}=176)\end{array}$} & \multicolumn{2}{|c|}{$\begin{array}{l}\text { Estudo de consistência interna e validade de } \\
\text { construto }(n=1.321)\end{array}$} \\
\hline & $\mathbf{n}$ & $\%$ & $\mathbf{n}$ & $\%$ \\
\hline \multicolumn{5}{|l|}{ Sexo } \\
\hline Masculino & 71 & 40,3 & 612 & 46,3 \\
\hline Feminino & 105 & 59,7 & 709 & 53,7 \\
\hline \multicolumn{5}{|l|}{ Faixa etária (anos) * } \\
\hline $10-12$ & 86 & 64,7 & 752 & 56,9 \\
\hline $13-15 * *$ & 47 & 35,3 & 569 & 43,1 \\
\hline \multicolumn{5}{|l|}{ 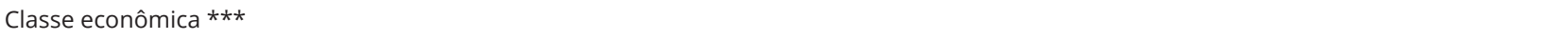 } \\
\hline Classe A/B & 52 & 31,7 & 454 & 34,4 \\
\hline Classe C/D/E & 114 & 68,3 & 867 & 65,6 \\
\hline QVRS & Média & DP & Média & DP \\
\hline Domínio 1 & 76,4 & 15,2 & 77,7 & 14,1 \\
\hline Domínio 2 & 83,5 & 14,1 & 85,7 & 12,1 \\
\hline Domínio 3 & 75,6 & 14,4 & 77,0 & 15,8 \\
\hline Domínio 4 & 80,7 & 18,1 & 81,9 & 16,5 \\
\hline Domínio 5 & 84,0 & 14,9 & 85,5 & 14,5 \\
\hline Escore geral de QVRS & 79,4 & 11,4 & 81,3 & 10,5 \\
\hline
\end{tabular}

Domínio 1: saúde e bem-estar; Domínio 2: bem-estar psicológico; Domínio 3: autonomia e relação com os pais;

Domínio 4: suporte social e grupo de pares; Domínio 5: ambiente escolar; DP: desvio padrão; QVRS: qualidade de vida relaciona à saúde.

* Variável com 43 não respostas para a amostra do estudo de reprodutibilidade;

** Para o estudo de consistência interna e validade a idade dos adolescentes variou de 10-14 anos;

*** Variável com 10 não respostas para a amostra do estudo de reprodutibilidade.

\section{Tabela 2}

Coeficiente de correlação intraclasse (CCI) para a medida de reprodutibilidade do questionário KIDSCREEN-27 em adolescentes de João Pessoa, Paraíba, Brasil, 2013.

\begin{tabular}{|c|c|c|c|c|c|c|}
\hline Variáveis & $\begin{array}{l}\text { Domínio } 1 \\
\text { CCI (IC95\%) }\end{array}$ & $\begin{array}{l}\text { Domínio } 2 \\
\text { CCI (IC95\%) }\end{array}$ & $\begin{array}{l}\text { Domínio } 3 \\
\text { CCI (IC95\%) }\end{array}$ & $\begin{array}{l}\text { Domínio } 4 \\
\text { CCI (IC95\%) }\end{array}$ & $\begin{array}{l}\text { Domínio } 5 \\
\text { CCI (IC95\%) }\end{array}$ & $\begin{array}{c}\text { Escore QVRS } \\
\text { CCI (IC95\%) }\end{array}$ \\
\hline Todos & $0,75(0,67-0,82)$ & $0,81(0,74-0,86)$ & $0,85(0,80-0,89)$ & $0,85(0,80-0,89)$ & $0,89(0,85-0,92)$ & $0,90(0,87-0,93)$ \\
\hline \multicolumn{7}{|l|}{ Sexo } \\
\hline Masculino & $0,78(0,64-0,86)$ & $0,77(0,70-0,80)$ & $0,90(0,85-0,94)$ & $0,86(0,76-0,91)$ & $0,95(0,92-0,97)$ & $0,92(0,87-0,95)$ \\
\hline Feminino & $0,71(0,57-0,81)$ & $0,86(0,79-0,90)$ & $0,83(0,74-0,87)$ & $0,86(0,79-0,91)$ & $0,87(0,80-0,91)$ & $0,91(0,85-0,94)$ \\
\hline \multicolumn{7}{|c|}{ Faixa etária (anos) } \\
\hline $10-12$ & $0,77(0,67-0,84)$ & $0,82(0,74-0,87)$ & $0,85(0,75-0,89)$ & $0,87(0,80-0,91)$ & $0,92(0,89-0,94)$ & $0,91(0,88-0,95)$ \\
\hline $13-15$ & $0,70(0,64-0,83)$ & $0,82(0,66-0,90)$ & $0,89(0,79-0,94)$ & $0,85(0,72-0,91)$ & $0,84(0,72-0,91)$ & $0,89(0,80-0,94)$ \\
\hline \multicolumn{7}{|c|}{ Classe econômica } \\
\hline $\mathrm{A} / \mathrm{B}$ & $0,85(0,74-0,92)$ & $0,89(0,80-0,94)$ & $0,93(0,88-0,96)$ & $0,84(0,71-0,91)$ & $0,92(0,87-0,96)$ & $0,96(0,92-0,98)$ \\
\hline$C / D / E$ & $0,72(0,62-0,78)$ & $0,75(0,63-0,83)$ & $0,81(0,71-0,87)$ & $0,87(0,80-0,91)$ & $0,90(0,86-0,93)$ & $0,87(0,80-0,92)$ \\
\hline
\end{tabular}

Domínio 1: saúde e bem-estar; Domínio 2: bem-estar psicológico; Domínio 3: autonomia e relação com os pais; Domínio 4: suporte social e grupo de pares; Domínio 5: ambiente escolar; IC95\%: intervalo de 95\% de confiança; QVRS: qualidade de vida relaciona à saúde. 


\section{Figure 1}

Resultados na análise fatorial confirmatória do questionário para avaliar qualidade de vida relacionada à saúde - KIDSCREEN-27 em adolescentes de João Pessoa, Paraíba, Brasil, para o modelo sem (2014, ( $\chi^{2}$ [df] = 866,3 [311], $p<0,001 ; \chi^{2} / \mathrm{df}=2,79 ; \mathrm{RMR}=0,035 ; \mathrm{RMSEA}=0,037$ [IC90\%: 0,034-0,040]; $\mathrm{AGFI}=0,941 ; \mathrm{GFI}=0,951 ; \mathrm{CFI}=0,908)$ e com a presença de covariâncias entres os erros de medida $\left(\chi^{2}[\mathrm{df}]=866,3[311], \mathrm{p}<0,001 ; \chi^{2} / \mathrm{df}=2,79 ; \mathrm{RMR}=\right.$ 0,035; RMSEA = 0,037 [IC90\%: 0,034-0,040]; GFI = 0,951; AGFI = 0,941; CFI = 0,908; TLI = 0,901) de alguns itens do D2 e D3.

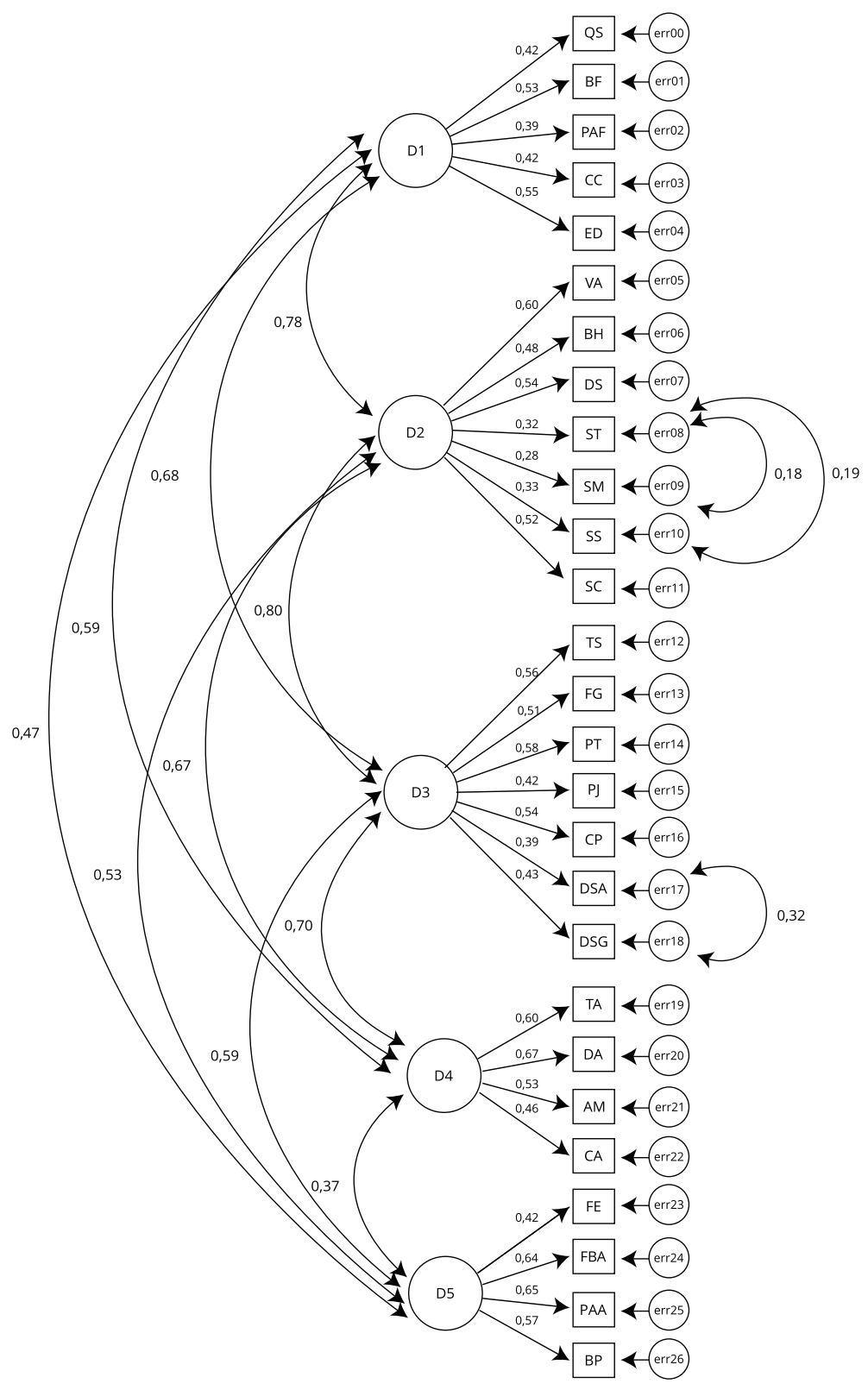

AGFI: Adjusted Goodness of Fit Index; AM: amizade mútua; BF: bem-estar físico; BH: bom humor; BP: boa relação com os professores; CA: confiou nos amigos; CC: capaz de correr; CFI: Comparative Fit Index; CP: conversou com os seus pais; DA: divertiu-se com os amigos; df: degrees of freedom; Domínio 1: saúde e bem-estar; Domínio 2: bem-estar psicológico; Domínio 3: autonomia e relação com os pais; Domínio 4: suporte social e grupo de pares; Domínio 5: ambiente escolar; DS: divertiu-se; DSA: dinheiro suficiente para gastos com os amigos; DSG: dinheiro suficiente para seus gastos; ED: energia e disposição; FBA: foi um bom aluno; FE: feliz na escola; FG: fez o que gosta; IC90\%: intervalo de 90\% de confiança; GFI: Goodness of Fit Index; PAA: prestou atenção nas aulas; PAF: praticou atividade física; PJ: pais trataram você de forma justa; PT: pais tiveram tempo suficiente para você; QS: qualidade de sua saúde; QVRS: qualidade de vida relaciona à saúde; RMR: Root Mean Square Residual; RMSEA: Root Mean Square of Approximation; SC: sentiu-se contente com o jeito de ser; SM: sentiu-se mal; SS: sentiu-se sozinho; ST: sentiu-se triste; TA: tempo suficiente para ficar com os amigos; TS: tempo suficiente; VA: vida agradável. 
Tabela 3

Resultados da análise fatorial confirmatória (parâmetros da qualidade de ajuste dos modelos) para avaliação da validade de construto do questionário para avaliar a qualidade de vida relacionada à saúde. KIDSCREEN-27 em adolescentes, João Pessoa, Paraíba, Brasil, 2014.

\begin{tabular}{|c|c|c|c|c|c|c|c|c|}
\hline Variáveis & $\chi^{2}(\mathrm{df})$ & Valor de $p$ & $\chi^{2 / d f}$ & RMR & RMSEA (IC90\%) & AGFI & GFI & CFI \\
\hline \multicolumn{9}{|l|}{ Sexo } \\
\hline Masculino & $617,1(311)$ & $<0,001$ & 1,98 & 0,035 & $0,040(0,035-0,045)$ & 0,915 & 0,930 & 0,879 \\
\hline Feminino & $669,9(311)$ & $<0,001$ & 2,15 & 0,044 & $0,040(0,036-0,045)$ & 0,918 & 0,933 & 0,896 \\
\hline \multicolumn{9}{|c|}{ Faixa etária (anos) } \\
\hline $10-12$ & $620,0(311)$ & $<0,001$ & 1,99 & 0,038 & $0,043(0,039-0,047)$ & 0,916 & 0,930 & 0,869 \\
\hline $13-14$ & $712,8(311)$ & $<0,001$ & 2,29 & 0,034 & $0,036(0,032-0,041)$ & 0,929 & 0,941 & 0,906 \\
\hline \multicolumn{9}{|c|}{ Classe econômica } \\
\hline $\mathrm{A} / \mathrm{B}$ & $574,2(311)$ & $<0,001$ & 1,85 & 0,045 & $0,046(0,040-0,052)$ & 0,902 & 0,902 & 0,864 \\
\hline $\mathrm{C} / \mathrm{D} / \mathrm{E}$ & $642,2(311)$ & $<0,001$ & 2,06 & 0,040 & $0,037(0,033-0,041)$ & 0,927 & 0,940 & 0,907 \\
\hline
\end{tabular}

AGFI: Adjusted Goodness of Fit Index; CFI: Comparative Fit Index; df: degrees of freedom; GFI: Goodness of Fit Index; IC90\%: intervalo de 90\% de confiança; RMR: Root Mean Square Residual; RMSEA: Root Mean Square of Approximation.

\section{Tabela 4}

Consistência interna do questionário KIDSCREEN-27 em adolescentes de João Pessoa, Paraíba, Brasil, 2013.

\begin{tabular}{|c|c|c|c|c|c|c|}
\hline Variáveis & $\begin{array}{c}\text { Domínio } 1 \\
\text { IFC }\end{array}$ & $\begin{array}{c}\text { Domínio } 2 \\
\text { IFC }\end{array}$ & $\begin{array}{c}\text { Domínio } 3 \\
\text { IFC }\end{array}$ & $\begin{array}{c}\text { Domínio } 4 \\
\text { IFC }\end{array}$ & $\begin{array}{c}\text { Domínio } 5 \\
\text { IFC }\end{array}$ & $\begin{array}{c}\text { Questionário } \\
\text { IFC }\end{array}$ \\
\hline Todos & 0,65 & 0,65 & 0,70 & 0,65 & 0,65 & 0,90 \\
\hline \multicolumn{7}{|l|}{ Sexo } \\
\hline Masculino & 0,64 & 0,68 & 0,67 & 0,63 & 0,67 & 0,89 \\
\hline Feminino & 0,61 & 0,68 & 0,72 & 0,66 & 0,66 & 0,90 \\
\hline \multicolumn{7}{|c|}{ Faixa etária (anos) } \\
\hline $10-12$ & 0,67 & 0,63 & 0,70 & 0,66 & 0,66 & 0,90 \\
\hline $13-15$ & 0,62 & 0,66 & 0,71 & 0,64 & 0,66 & 0,89 \\
\hline \multicolumn{7}{|c|}{ Classe econômica } \\
\hline $\mathrm{A} / \mathrm{B}$ & 0,65 & 0,69 & 0,67 & 0,64 & 0,66 & 0,90 \\
\hline$C / D / E$ & 0,65 & 0,65 & 0,71 & 0,65 & 0,66 & 0,90 \\
\hline
\end{tabular}

Domínio 1: saúde e bem-estar; Domínio 2: bem-estar psicológico; Domínio 3: autonomia e relação com os pais; Domínio 4: suporte social e grupo de pares; Domínio 5: ambiente escolar; IFC: índice de fidedignidade combinada $=\left\{\left[\left(\sum \text { das cargas fatoriais }\right)^{2 /(}\left(\sum \text { das cargas fatoriais }\right)^{2}\right]+\right.$

[ $\Sigma\left(1\right.$ - cargas fatoriais $\left.\left.{ }^{2}\right)\right]$

Noruega de 10 anos de idade (CCI: 0,71 a 0,81), observaram níveis de reprodutibilidade inferiores aos do presente trabalho (CCI: 0,75 a 0,89), enquanto que nos estudos de Quintero et al. 13 (adolescentes da Colômbia de 8-18 anos - CCI superiores a 0,87) e Ng et al. 28 (adolescentes da China com média de idade de 12 anos - CCI: 0,78 a 0,86) os valores foram similares.

Diferenças nos valores do CCI para o KIDSCREEN-27 entre os estudos podem ser atribuídas ao número de dias utilizados entre as réplicas de aplicação do instrumento, à forma de aplicação (autoadministrado $v s$. entrevista) e à faixa etária dos adolescentes.

No presente trabalho, o questionário foi reaplicado com intervalo de dois dias. Isso permitiu que os adolescentes recordassem sobre a mesma semana para responder as suas questões. Por outro lado, praticamente todos os estudos que analisaram a reprodutibilidade usaram um período de sete dias ou mais entre as réplicas de aplicação, exceto o que foi realizado por Andersen et al. 27 (intervalo de 12 a 
24 horas). Utilizar períodos prolongados de tempo entre as réplicas de aplicação - por exemplo, uma semana ou mais - leva os adolescentes a recordarem o que ocorreu com eles em períodos diferentes. Com isso, possíveis alterações nas percepções dos indicadores mensurados entre as aplicações do instrumento, decorrentes de mudanças nas vidas dos adolescentes, serão tratadas como erro de medida, podendo resultar em menores níveis de reprodutibilidade 19.

O KIDSCREEN-27 foi desenvolvido para ser aplicado na forma autoadministrada ou respondida pelos pais ou responsáveis (proxy-report). Nos estudos de reprodutibilidade, esse instrumento foi aplicado na forma autoadministrada. No presente estudo, dado seu público se tratar de adolescentes jovens e de baixa condição econômica, optou-se por aplicá-lo na forma de entrevista face a face. $\mathrm{O}$ uso de um intervalo de tempo curto entre as réplicas de aplicação, associado à aplicação na forma de entrevista, poderia explicar os valores ligeiramente mais elevados para CCI no presente trabalho. Apesar de essa forma de aplicação demandar maior quantidade de tempo e de recursos humanos, ela tende a produzir resultados mais precisos.

Em relação à idade, apesar de os adolescentes mais jovens apresentarem maiores dificuldades para interpretar e responder questões sobre aspectos subjetivos e a percepção sobre a qualidade de vida mudar com a idade 1,29,30, a reprodutibilidade do KIDSCREEN-27 para os adolescentes tem sido similar entre os adolescentes de diferentes faixas etárias 7,13,27,28. Esse resultado foi confirmado no presente estudo.

A maioria dos itens de cada domínio apresentou valores acima de 0,40 para a CF, exceto para um item do domínio 1 [D1] - bem-estar físico ("praticou atividades físicas", $\mathrm{CF}=0,39$ ), três itens do domínio 2 [D2] - bem-estar psicológico ("você se sentiu triste", "você se sentiu tão mal que não queria fazer nada", "você se sentiu sozinho"), com CF variando de 0,28 a 0,39, e um no domínio 3 [D3] - pais e suporte social ("teve dinheiro suficiente para fazer as mesmas coisas que seus amigos fizeram", $\mathrm{CF}=0,39$ ).

As CF identificadas neste trabalho, em geral, foram ligeiramente inferiores às de estudos com adolescentes de 13 países da Europa 31 (domínio 1: 0,51 a 0,76; domínio 2: 0,56 a 0,74; domínio 3: 0,53 a 0,72; domínio 4: 0,50 a 0,80; domínio 5: 0,66 a 0,76), da Ásia 28 (0,68 a 0,95) e de países da América do Sul (domínio 1: 0,60 a 0,74; domínio 2: 0,37 a 0,76; domínio 3: 0,41 a 0,80; domínio 4: 0,66 a 0,85; domínio 5: 0,58 a 0,75) 13,14,32.

A presença de CF mais baixas no KIDSCREEN-27 entre os adolescentes brasileiros pode ser explicada pela população-alvo do estudo: faixa etária (de 10 a 14 anos, com 56,9\% de $10-12$ anos) e condição econômica restrita (a maioria de famílias de baixa condição econômica: 66,5\% das classes econômicas $\mathrm{C} / \mathrm{D} / \mathrm{E}$ ). A homogeneidade da amostra em relação a esses aspectos e aos níveis de qualidade de vida relacionada à saúde pode ter contribuído para as correlações baixas entre os itens e os seus domínios, produzindo cargas fatoriais mais baixas ${ }^{30}$. Adolescentes mais jovens e de baixa condição socioeconômica apresentaram menores níveis de qualidade de vida relacionada à saúde ${ }^{29} \mathrm{e} \mathrm{de} \mathrm{com-}$ preensão das perguntas dos questionários 33,34 . Outras explicações para esses resultados: a formulação dos itens (alguns apresentam termos que podem ser entendidos como sendo sinônimos) e uma possível inadequação de alguns para avaliar a qualidade de vida no grupo estudado, e distribuição dos itens dentro de cada domínio. É preciso realizar estudos com adolescentes brasileiros de diferentes faixas etárias e condições socioeconômicas para esclarecer esses aspectos.

Uma maior quantidade de itens com CF bem abaixo de 0,40 no domínio bem-estar psicológico pode ser explicada pelo fato de que estes itens eram provenientes de diferentes domínios do KIDSCREEN-52 7,8; com isto podem não estar relacionados ao domínio em questão na versão com 27 itens. Ng et al. 28 e Shannon et al. 25 testaram o KIDSCREEN-27 com sete domínios, sendo adicionados os domínios "humor e emoções" e "recursos financeiros", formados por três itens que faziam parte do domínio "bem-estar psicológico" "você se sentiu triste", "você se sentiu tão mal que não queria fazer nada", "você se sentiu sozinho") e dois do domínio "pais e suporte social" ("teve dinheiro suficiente para fazer as mesmas coisas que seus amigos fizeram" e "teve dinheiro suficiente para os seus gastos”), respectivamente. Nesses estudos, as CF desses itens foram superiores a 0,50.

Deve-se levar em conta que a maioria dos adolescentes estudados pode ter considerado que esses três itens mensuravam os mesmos traços relacionados à qualidade de vida relacionada à saúde, sugerindo possíveis problemas na construção dos mesmos. Esse fenômeno pode ter sido acentuado no presente estudo, tendo em vista que a maioria dos participantes tinha até 12 anos de idade e pertencia às classes econômicas baixas. Shannon et al. 25 também identificaram CF mais baixas para 
esses itens em jovens de 8-9 anos de idade e de baixo nível socioeconômico, o que não foi encontrado em estudos com adolescentes de faixa etária mais ampla (8-18 anos) e de diferentes condições socioeconômicas $2,7,9,14,31$.

A utilização de termos negativos na construção dos três itens do domínio "bem-estar psicológico" pode ser outra fonte de explicação para esses resultados. Adolescentes jovens têm dificuldades para entender itens negativos em escalas de medida 35 , e o seu uso pode resultar em menores níveis de precisão 36,37 .

Embora não haja consenso sobre o valor mínimo de uma CF para ser considerado aceitável, optou-se por não excluir esses cinco itens do questionário. Primeiro porque, com a simulação da referente exclusão, a qualidade de ajuste do modelo piorou (0,045 a 0,052 para o RMR e o RMSEA; 0,86 a 0,88 para AGF e GFI; 0,87 a 0,89 para CFI, 0,832 a 0,845 para o TLI) comparativamente à observada no modelo com todos os itens. Segundo, CF próximas de 0,30 têm sido consideradas aceitáveis em estudos com grandes amostras 21. Porém, é preciso avaliar em futuros trabalhos a adequação desses itens nos domínios do KIDSCREEN-27.

Em relação ao número de domínios e seus respectivos itens, os resultados deste estudo confirmaram a composição do modelo original do KIDSCREEN-27. Os índices de qualidade de ajuste do modelo foram similares aos observados em países da Europa 25,27,31, da América do Sul (Chile) 12, e superiores aos de estudos do Chile 32 , Colômbia 13 e China 28.

A melhoria observada neste trabalho na qualidade do ajuste do modelo com a inclusão de covariâncias entre os erros (resíduos) de três itens do domínio 2 - bem-estar psicológico - e entre dois itens foi verificada por Andersen et al. 27 em adolescentes de 10 anos de idade. Normalmente, recomenda-se a exclusão dos itens com covariâncias entre os erros de medida. Entretanto, os indicadores de qualidade de ajuste do modelo sem esses itens foram bem inferiores (0,045 a 0,052 para RMR e RMSEA; 0,86 a 0,88 para AGF e GFI; 0,87 a 0,89 para CFI, 0,852 a 0,854 para TLI) aos que os incluíram - dados não apresentados em tabelas.

A presença de covariância entre os erros desses itens pode estar relacionada à semelhança da formulação desses itens, de modo que, possivelmente, os adolescentes não tenham discernido adequadamente os seus respectivos conteúdos. Não se pode descartar a possibilidade de eles estarem compartilhando uma ou mais causas comuns e/ou serem inadequados para representar os domínios nos quais estão inseridos. Shannon et al. 25 e Ng et al. 28 verificaram que o modelo do KIDSCREEN-27 com esses itens alocados em dois novos domínios resultou em melhores níveis de ajuste, comparativamente ao modelo original.

Uma análise complementar foi realizada considerando a presença de sete domínios, conforme sugerido por Shannon et al. 25 e Ng et al. 28 , mas os resultados do presente trabalho $\left(\chi^{2}[\mathrm{df}]=786,8\right.$ [303], $\mathrm{p}<0,001 ; \chi^{2} / \mathrm{df}=2,54 ; \mathrm{RMR}=0,045 ; \mathrm{RMSEA}=0,051$ [IC90\%: 0,043-0,061]; GFI = 0,895; $\mathrm{AGFI}=0,910 ; \mathrm{CFI}=0,808$; TLI = 0,857) não sustentaram a hipótese de que o KIDSCREEN-27 com sete domínios é mais adequado para representar o construto qualidade de vida relacionada à saúde, comparativamente ao modelo original, que contém cinco domínios. É preciso analisar o referencial teórico que determina quantos domínios e quais itens devem saturar em cada um deles para o KIDSCREEN-27, bem como o quanto esse modelo é adequado para adolescentes de países com rendas média/baixa e baixa, e com características socioculturais distintas daquelas encontradas na região em que ele foi desenvolvido e testado.

Um achado importante deste estudo foi ter identificado valores similares para os índices de qualidade de ajuste do modelo do KIDSCREEN-27 nos diferentes subgrupos analisados. Isso sugere que esse instrumento mensura a qualidade de vida relacionada à saúde de maneira similar em adolescentes com diferentes características sociodemográficas (sexo, faixa etária e classe econômica).

Em relação à consistência interna, o KIDSCREEN-27 alcançou valores adequados de IFC (IFC = 0,90), mas ligeiramente inferiores a 0,70 nos domínios, exceto no domínio 3 - pais e suporte social $(\mathrm{IFC}=0,70)$. Não foi identificada qualquer melhoria nos níveis de consistência interna em caso de exclusão de algum item. Ao se considerar a medida do $\alpha$, verificou-se que a consistência interna do instrumento foi ligeiramente inferior à observada pela medida do IFC. $\mathrm{O} \alpha$ subestima a consistência interna quando se utiliza a análise fatorial confirmatória e o instrumento tem mais de dois domínios 26. Valores de indicadores de consistência interna ( $\alpha$ e IFC) iguais ou superiores a 0,60 são considerados aceitáveis 38 . 
Os valores para a medida de consistência interna neste trabalho (IFC variou de 0,65 a 0,70 ) foram inferiores aos encontrados em estudos com adolescentes de países da Europa, conforme descrito por Raviens et al. 7 ( $\alpha$ de 0,80 a 0,84), Robitail et al. 31 ( $\alpha$ de 0,78 a 0,84) e Andersen et al. 27 ( $\alpha$ de 0,77 a 0,82), da América do Sul, com valores de $\alpha$ variando de 0,73 a 0,7532 ou superiores a 0,75 12, e da Ásia ( $\alpha$ de 0,84 a 0,91); e similares aos do estudo de Shannon et al. 25 com adolescentes da Irlanda ( $\alpha$ de 0,65 a 0,74). Valores mais baixos de consistência interna nos adolescentes brasileiros podem ser decorrentes da amplitude da idade (56,7\% de 10-12 anos), da amostra e da utilização do IFC para estimar a consistência interna. Nos trabalhos analisados, a consistência interna foi avaliada pelo $\alpha$, e a maioria envolveu adolescentes foi com faixa etária mais ampla. O IFC apresenta valores inferiores aos do $\alpha$ para um mesmo instrumento, e a variabilidade das respostas interfere na proporção da variância explicada por cada domínio do instrumento 39. É possível que entre os adolescentes mais jovens certos itens do KIDSCREEN-27 não sejam tão relevantes para a sua qualidade de vida relacionada à saúde; eles podem ter dificuldades para discernir adequadamente o conteúdo de algumas questões (por exemplo, alguns itens dos domínios 2 e 3 ) e emitir uma resposta que melhor represente a sua percepção para estes aspectos 30 .

Este estudo apresentou pontos fortes que devem ser destacados: usou uma amostra representativa de escolares de bairros com diferentes condições socioeconômicas e estruturais, com poder adequado para testar as hipóteses em estudo; reprodutibilidade, consistência interna e validade de construto foram avaliadas em diferentes subgrupos.

As principais limitações deste trabalho incluem: não avaliou a validade concorrente do questionário; o estudo foi realizado com adolescentes de faixa etária restrita (10-15 anos) e que estudavam em escolas da rede pública, limitando a generalização dos achados para outras faixas etárias e, possivelmente, para os que estudavam na rede privada de ensino. $\mathrm{O}$ fato de o questionário ter sido aplicado por entrevista "face a face" limita a generalização dos resultados dos níveis de reprodutibilidade, consistência interna e validade de construto para a aplicação por meio de autopreenchimento.

O KIDSCREEN-27 alcançou níveis satisfatórios de reprodutibilidade, consistência interna e validade de construto, podendo ser usado para mensurar a qualidade de vida relacionada à saúde em adolescentes na faixa etária de 10-15 anos e com características socioeconômicas semelhantes aos analisados neste trabalho. É necessário realizar estudos para avaliar reprodutibilidade, consistência interna e validade do KIDSCREEN-27 e sua invariância fatorial em adolescentes mais velhos ( $>15$ anos de idade) e/ou de faixa etária mais ampla (10-19 anos) e de várias regiões do Brasil.

\section{Colaboradores}

J. C. Farias Júnior participou de todas as etapas do estudo, incluindo concepção do projeto de pesquisa e do artigo, coleta e análise dos dados, redação e revisão do manuscrito. M. R. Loch e F. E. L. L. Ferreira contribuíram na redação e revisão crítica do manuscrito. A. J. Lima Neto colaborou na redação do manuscrito. J. M. Sales participou da concepção do artigo, coleta de dados e da redação do manuscrito.

\section{Agradecimentos}

Ao CNPq (Conselho Nacional de Desenvolvimento Científico e Tecnológico) e à FAPESQ (Fundação de Apoio à Pesquisa do Estado da Paraíba) pela concessão de recursos financeiros por meio do Edital Universal e PPSUS (Programa Pesquisa para o Sistema Único de Saúde), respectivamente, para o Estudo LONCAAFS. 


\section{Referências}

1. Centers for Disease Control and Prevention. Measuring healthy days: population assessment of health-related quality of life. Atlanta: Centers for Disease Control and Prevention; 2000.

2. Soares AHR, Martins AJ, Lopes MCB, Britto JAA, Oliveira CQ, Moreira MCN. Qualidade de vida de crianças e adolescentes: uma revisão bibliográfica. Ciênc Saúde Coletiva 2011; 16:3197-206.

3. von Rueden U, Gosch A, Rajmil L, Bisegger C, Ravens-Sieberer U. Socioeconomic determinants of health related quality of life in childhood and adolescence: results from a European study. J Epidemiol Community Health 2006; 60:130-5.

4. Barros LP, Gropo LN, Petribú K, Colares V. Avaliação da qualidade de vida em adolescentes - revisão da literatura. J Bras Psiquiatr 2008; 57:212-7.

5. Solans M, Pane S, Estrada M-D, Serra-Sutton V, Berra S, Herdman M, et al. Health-related quality of life measurement in children and adolescents: a systematic review of generic and disease-specific instruments. Value Health 2008; 11:742-64.

6. Kenzik KM, Tuli SY, Revicki DA, Shenkman EA, Huang I-C. Comparison of 4 pediatric health-related quality-of-life instruments: a study on a medicaid population. Med Decis Making 2014; 34:590-602.

7. Ravens-Sieberer U, Auquier P, Erhart M, Gosch A, Rajmil L, Bruil J, et al. The KIDSCREEN-27 quality of life measure for children and adolescents: psychometric results from a cross-cultural survey in 13 European countries. Qual Life Res 2007; 16:1347-56.

8. Ravens-Sieberer U, Herdman M, Devine J, Otto C, Bullinger M, Rose M, et al. The European KIDSCREEN approach to measure quality of life and well-being in children: development, current application, and future advances. Qual Life Res 2014; 23:791-803.
9. Stevanovic D, Jafari P. A cross-cultural study to assess measurement invariance of the KIDSCREEN-27 questionnaire across Serbian and Iranian children and adolescents. Qual Life Res 2015; 24:223-30.

10. Guedes DP, Guedes JERP. Tradução, adaptação transcultural e propriedades psicométricas do KIDSCREEN-52 para a população brasileira. Rev Paul Pediatr 2011; 29:364-71.

11. Bagheri Z, Jafari P, Tashakor E, Kouhpayeh A, Riazi H. Assessing whether measurement invariance of the KIDSCREEN-27 across childparent dyad depends on the child gender: a multiple group confirmatory factor analysis. Glob J Health Sci 2014; 6:142-53.

12. Molina T, Montaño R, González E, Sepúlveda R, Hidalgo-Rasmussen C, Martínez V, et al. Propiedades psicométricas del cuestionario de calidad de vida relacionada con la salud KIDSCREEN-27 en adolescentes chilenos. Rev Med Chil 2014; 142:1415-21.

13. Quintero CA, Lugo LH, García HI, Sánchez A. Validación del cuestionario KIDSCREEN-27 de calidad de vida relacionada con la salud en niños y adolescentes de Medellín, Colombia. Rev Colomb Psiquiatr 2011; 40:470-87.

14. Vélez CM, Lugo LH, García HI. Validez y confiabilidad del cuestionario de calidad de vida KIDSCREEN-27 versión padres, en Medellín, Colombia. Rev Colomb Psiquiat 2012; 41:588605.

15. Vingilis ER, Wade TJ, Seeley JS. Predictors of adolescent self-rated health: analysis of the national population health survey. Can J Public Health 2002; 93:193-7.

16. Guillemin F. Cross-cultural adaptation of health-related quality of life measures: literature review and proposed guidelines. J Clin Epidemiol 1993; 46:1417-32. 
17. Epstein J, Santo RM, Guillemin F. A review of guidelines for cross-cultural adaptation of questionnaires could not bring out a consensus. J Clin Epidemiol 2015; 68:435-41.

18. Associação Brasileira de Empresas de Pesquisa. Critério de classificação econômica Brasil. http://www.abep.org/novo/Default.aspx (acessado em 20/Mai/2013).

19. Kimberlin CL, Winterstein AG. Validity and reliability of measurement instruments used in research. Am J Health Syst Pharm 2008; 65:2276-84.

20. Pasquali L. Psicometria. Rev Esc Enferm USP 2009; 43(Esp):992-9.

21. Kline RM. Principles and practice of structural equation modeling. 2nd Ed. New York: Guild Ford Press; 2005.

22. Chinapaio MJM, Mokkink LB, van Poppel MNM, van Mechelen W, Terwee CB. Physical activity questionnaires for youth: a systematic review of measurement properties. Sports Med 2010; 40:539-63.

23. Lin TI. Maximum likelihood estimation for multivariate skew normal mixture models. J Multivar Anal 2009; 100:257-65.

24. Byrne BM. Structural equation modeling with AMOS, EQS, and LISREL: comparative approaches to testing for the factorial validity of a measuring instrument. International Journal of Testing 2011; 1:55-86.

25. Shannon S, Breslin G, Fitzpatrick B, Hanna $D$. Testing the psychometric properties of Kidscreen-27 with Irish children of low socio-economic status. Qual Life Res 2017; 26:1081-9.

26. Peterson RA, Kim Y. On the relationship between coefficient alpha and composite reliability. J Appl Psychol 2013; 98:194-8.

27. Andersen JR. Pyschometric properties of the Norweigian version of the KIDSCREEN-27 questionnaire. Health Qual Life Outcomes 2016; 14:58.

28. Ng JY, Burnett A, Ha AS, Sum KW. Psychometric properties of the Chinese (Cantonese) versions of the KIDSCREEN health-related quality of life questionnaire. Qual Life Res 2015; 24:2415-2.

29. Gaspar T, Matos MG, Pais R, Jose L, Leal I, Ferreira A. Health-related quality of life in children and adolescents and associated factors. J Cogn Behav Psychother 2009; 9:33-48.
30. Anderson JC, Gerbing DW. The effect of sampling error on convergence, improper solutions, and goodness-of-fit indices for maximum likelihood confirmatory factor analysis. Psychometrika 1984; 49:155-73.

31. Robitail S, Ravens-Sieberer U, Simeoni M-C, Rajmil L, Bruil J, Power M, et al. Testing the structural and cross-cultural validity of the KIDSCREEN-27 quality of life questionnaire. Qual Life Res 2007; 16:1335-45.

32. Urzúa A, Cortés E, Vega S, Prieto L, Tapia K. Propiedades psicométricas del cuestionario de auto reporte de la calidad de vida KIDSCREEN-27 en adolescentes chilenos. Ter Psicol 2009; 27:83-92.

33. Eiser C, Morse R. Can parents rate their child's health-related quality of life? Results of a systematic review. Qual Life Res 2001; 10:347-57.

34. Reichenheim ME, Moraes CL. Operacionalização de adaptação transcultural de instrumentos de aferição usados em epidemiologia. Rev Saúde Pública 2007; 41:665-73.

35. Benson J, Hocevar D. The impact of item phrasing on the validity of attitude scales for elementary school children. J Educ Meas 1985; 22:231-40.

36. Roszkowski MJ, Soven M. Shifting gears: consequences of including two negatively worded items in the middle of a positively worded questionnaire. Assess Eval High Educ 2010; 35:113-30.

37. Salazar MS. The dilemma of combining positive and negative items in scales. Psicothema $2015 ; 37: 192-9$

38. Loewenthal KM. An introduction to psychological tests and scales. 2nd Ed. Hove: Psychology Press; 2001.

39. Dunn TJ, Baguley T, Brunsden V. From alpha to omega: a practical solution to the pervasive problem of internal consistency estimation. $\mathrm{Br}$ J Psychol 2014; 105:399-412. 


\section{Abstract}

The objective of this two-part study was to estimate the reproducibility, internal consistency, and construct validity of KIDSCREEN-27, a questionnaire to measure health-related quality of life, in Brazilian adolescents. One study component estimated reproducibility (176 adolescents, $59.7 \%$ females, $64.7 \% 10$ to 12 years of age), and another estimated internal consistency and validity (1,321 adolescents, 53.7\% females, 56.9\% 10 to 12 years of age). The studies were conducted with adolescents of both sexes in public schools in the municipality of João Pessoa, Paraíba State, Brazil. KIDSCREEN-27 consists of 27 items distributed across five domains (physical well-being, 5 items; psychological well-being, 7 items; parents and social support, 7 items; autonomy and relationship with parents, 4 items; school environment, 4 items). Reproducibility was estimated by intraclass correlation coefficient (ICC). Confirmatory factor analysis was used to assess construct validity, and composite reliability index (CRI) was used to verify the questionnaire's internal consistency. ICCs were greater than or equal to $0.70(0.70$ to 0.96). Factor loads were greater than 0.40 , except for five items ( 0.28 to 0.39 ). The model's goodnessof-fit indices were adequate $\left(\chi^{2} / d f=2.79 ; R M R\right.$ $=0.035 ; \mathrm{RMSEA}=0.037 ; \mathrm{GFI}=0.951 ; \mathrm{AGFI}$ $=0.941 ; C F I=0.908 ; T L I=0.901) . C R I$ varied from 0.65 to 0.70 in the domains and was 0.90 for the questionnaire. KIDSCREEN-27 reached satisfactory levels of reproducibility, internal consistency, and construct validity and can be used to assess health-related quality of life in Brazilian adolescents 10 to 15 years of age.

Surveys and Questionnaires; Reproducibility of Results; Quality of Life; Adolescent

\section{Resumen}

LEl objetivo de este estudio fue estimar reproducibilidad, consistencia interna y validez de constructo del KIDSCREEN-27, un cuestionario para medir la calidad de vida, relacionada con la salud, en adolescentes brasileños. Se realizó un estudio para estimar la reproducibilidad (176 adolescentes, 59,7\% del sexo femenino, 64,7\% de 10 a 12 años de edad) y otro para estimar la consistencia interna y la validez (1.321 adolescentes, $53,7 \%$ del sexo femenino, 56,9\% de 10 a 12 años de edad). Los estudios se realizaron con adolescentes de ambos sexos, de escuelas públicas, en el municipio de João Pessoa, Paraíba, Brasil. El KIDSCREEN-27 contiene 27 items distribuidos en cinco dominios (bienestar físico, 5 items; bienestar psicológico, 7 items; país y apoyo social, 7 items; autonomía $y$ relación con los padres, 4 items; ambiente escolar, 4 items). Para estimar la reproducibilidad, se recurrió al coeficiente de correlación intraclase - CCI. El análisis factorial confirmatorio se utilizó para evaluar la validez del constructo, y el indice de fiabilidad combinada (IFC) para verificar la consistencia interna del cuestionario. Los CCI fueron iguales o superiores a 0,70 (0,70 a 0,96). Las cargas factoriales fueron superiores a 0,40 , excepto en cinco items $(0,28$ a 0,39). Los indicadores de calidad de ajuste del modelo fueron adecuados $\left(\chi^{2} / d f=2,79 ; R M R=0,035 ; R M S E A=\right.$ 0,$037 ; G F I=0,951 ; A G F I=0,941 ; C F I=0,908$; $T L I=0,901)$. El IFC varió de 0,65 a 0,70 en los dominios y fue igual a 0,90 para el cuestionario. El KIDSCREEN-27 alcanzó niveles satisfactorios de reproducibilidad, consistencia interna y validez de construto, pudiendo ser utilizado para evaluar la calidad de vida relacionada con la salud en adolescentes de 10 a 15 años de edad.

Encuestas y Cuestionarios; Reproducibilidad de Resultados; Calidad de Vida; Adolescente
Recebido em 26/Jul/2016

Versão final reapresentada em 28/Mar/2017 Aprovado em 12/Mai/2017 